University of Wollongong

Research Online

Faculty of Engineering and Information

Faculty of Engineering and Information

Sciences - Papers: Part A

Sciences

$1-1-2016$

Correcting intensity loss errors in the absence of texture-free reference samples during pole figure measurement

Ahmed A. Saleh

University of Wollongong, asaleh@uow.edu.au

Quoc Viet Vu

University of Wollongong, qvv719@uowmail.edu.au

Azdiar A. Gazder

University of Wollongong, azdiar@uow.edu.au

Follow this and additional works at: https://ro.uow.edu.au/eispapers

Part of the Engineering Commons, and the Science and Technology Studies Commons

Research Online is the open access institutional repository for the University of Wollongong. For further information contact the UOW Library: research-pubs@uow.edu.au 


\title{
Correcting intensity loss errors in the absence of texture-free reference samples during pole figure measurement
}

\begin{abstract}
Even with the use of X-ray polycapillary lenses, sample tilting during pole figure measurement results in a decrease in the recorded X-ray intensity. The magnitude of this error is affected by the sample size and/or the finite detector size. These errors can be typically corrected by measuring the intensity loss as a function of the tilt angle using a texture-free reference sample (ideally made of the same alloy as the investigated material). Since texture-free reference samples are not readily available for all alloys, the present study employs an empirical procedure to estimate the correction curve for a particular experimental configuration. It involves the use of real texture-free reference samples that pre-exist in any $X$-ray diffraction laboratory to first establish the empirical correlations between X-ray intensity, sample tilt and their Bragg angles and thereafter generate correction curves for any Bragg angle. It will be shown that the empirically corrected textures are in very good agreement with the experimentally corrected ones.
\end{abstract}

\section{Keywords}

texture, free, reference, samples, correcting, during, pole, figure, measurement, errors, loss, intensity, absence

Disciplines

Engineering | Science and Technology Studies

\section{Publication Details}

Saleh, A. A., Vu, V. Q. \& Gazder, A. A. (2016). Correcting intensity loss errors in the absence of texture-free reference samples during pole figure measurement. Materials Characterization, 118 425-430.

This journal article is available at Research Online: https://ro.uow.edu.au/eispapers/6158 


\title{
Correcting intensity loss errors in the absence of texture-free reference samples during pole figure measurement
}

\author{
Ahmed A. Saleh ${ }^{1 *}$, Viet Q. Vuํㅜ, Azdiar A. Gazder² \\ ${ }^{1}$ School of Mechanical, Materials and Mechatronic Engineering, University of Wollongong, \\ New South Wales 2522, Australia \\ 2 Electron Microscopy Centre, University of Wollongong, New South Wales 2500, Australia
}

\begin{abstract}
Even with the use of X-ray polycapillary lenses, sample tilting during pole figure measurement results in a decrease in the recorded X-ray intensity. The magnitude of this error is affected by the sample size and/or the finite detector size. These errors can be typically corrected by measuring the intensity loss as a function of the tilt angle using a texture-free reference sample (ideally made of the same alloy as the investigated material). Since texture-free reference samples are not readily available for all alloys, the present study employs an empirical procedure to estimate the correction curve for a particular experimental configuration. It involves the use of real texture-free reference samples that pre-exist in any X-ray diffraction laboratory to first establish the empirical correlations between X-ray intensity, sample tilt and their Bragg angles and thereafter generate correction curves for any Bragg angle. It will be shown that the empirically corrected textures are in very good agreement with the experimentally corrected ones.
\end{abstract}

Keywords: texture-free reference sample, random sample, texture, pole figure, orientation distribution function

\section{* Corresponding author:}

Ahmed A. Saleh

School of Mechanical, Materials and Mechatronic Engineering University of Wollongong, New South Wales 2522, Australia Phone: +612 $42213034 \quad$ Fax: +612 $42213662 \quad$ e-mail: asaleh@uow.edu.au 


\section{Introduction}

The measurement of bulk crystallographic textures via X-ray diffraction begins with the collection of incomplete pole figures on the basis of specific crystal lattice planes recording intensities upon fulfilling Bragg's law. In this regard, the axes of a pole figure are defined by a macroscopic sample coordinate system whereas the pole figure itself is a contour plot stereogram of the angular distribution of crystallite plane normals within the sample [1].

The measurement of a single Bragg reflection (pole figure) on a goniometer involves mounting a sample on an Eulerian cradle and setting the X-ray source (or incident radiation) and detector to a particular peak position $(2 \theta$, where $\theta=$ Bragg angle). Thereafter, the systematic tilting $(\alpha)$ and rotation $(\beta)$ of the Eulerian cradle successively brings all possible lattice planes into the reflection condition and results in the collection of X-ray intensities for all angular orientations of the sample [1]. It follows that the recorded X-ray intensity for a given tilt and rotation angle is proportional to the volume fraction of particularly oriented crystallites in the sample.

During typical pole figure scans, the measured intensities are affected by absorption, background and defocusing errors. In the present study, correcting for absorption errors was unnecessary as the studied bulk sample is thicker than the penetration depth of the X-rays [2].

Background errors are caused by incoherent scattering events and fluorescence [1]. While the measured intensities ( $\mathrm{I}_{\text {meas }}$ ) are a function of both $\alpha$ and $\beta$ angles, the background intensities $\left(\mathrm{I}_{\mathrm{BG}}\right)$ are mainly affected by the $\alpha$-angle. Since the background intensities tend to remain stable for a given $\alpha$ angle, they are typically measured on either side of a Bragg peak, averaged and subtracted from the measured intensities [1, 3].

Defocusing errors are an instrumental aberration that manifest as a decrease in the measured intensity of a reflected peak due to the broadening of the diffracted beam with increasing sample tilt $[4]$.

Previous generation diffractometers employing crossed slits on the incident beam side were more prone to large defocusing errors. The limitations of the X-ray optics meant that defocusing errors were highly sensitive to: (i) variations in the irradiated area and broadening of the diffracted peaks with increasing tilt angle, (ii) the Bragg angle, (iii) the inaccurate positioning of the sample in the 
Eulerian cradle and/or, (iv) the incorrect alignment of the goniometer itself [3-7]. The conventional method to eliminate defocusing errors is via a correction function $\left(U_{I}\right)$ involving the pole figure scanning of a texture-free reference sample with a peak position and width close to that of the sample under investigation ${ }^{1}[1]$. Thus, for a given Bragg angle, the correction function is a measure of the change in the normalised intensity $\left(U_{I}=I_{\alpha=0-85^{\circ}} / I_{\alpha=0^{\circ}}\right)$ with tilt angle $[1,3,8]$. Alternatively, analytical methods that correct for defocusing in the classical Schultz reflection geometry with incident crossed slits have also been developed by a number of authors [3, 9-11].

On the other hand, modern diffractometers configured with the X-ray tube in point focus mode and a polycapillary lens on the incident beam side are less susceptible to large defocusing errors. This optical configuration: (i) reduces instrument aberrations by imposing a focused, (quasi-) parallel beam with a small angular divergence on the sample, (ii) returns higher diffracted intensities and, (iii) makes the overall measurement less sensitive to sample misalignments and peak broadening than traditional optics $[4,12,13]$.

While classical defocusing errors are minor when X-ray lens are used, other sources of geometrical error persist such that a decrease in measured intensity is returned when: (i) areas outside the sample surface are illuminated or, (ii) only a fraction of the diffracted beam is collected by the detector due to its finite size. Experimental and analytical methods have been developed to overcome these intensity losses $[4,13]$. In the case of analytical methods, they are based on the assumption that the reference and investigated samples are larger than the maximum irradiated area at the highest tilt angle [14].

Since the above conditionality cannot always be met in practice, a correction for the loss of intensity at high tilt angle still needs to be undertaken using texture-free reference samples. Given that texture-free reference samples are not readily available for all alloys, the present study details an empirical procedure to estimate the intensity loss correction required for a given goniometer configuration. Our method makes use of real texture-free reference samples that pre-exist in any X-ray diffraction laboratory to first establish the correlations between X-ray intensity and sample position in terms of $2 \theta, \alpha$ and $\beta$ angles and thereafter generate correction curves for any Bragg angle. It will be

\footnotetext{
${ }^{1}$ Ideally speaking, the texture-free reference samples should be made of the same alloy as the investigated material.
} 
shown that our correction curves are in very good agreement with experimental curves and that the former can be effectively used to correct incomplete pole figures when texture-free reference samples of the same alloy are absent.

\section{Experimental and analytical procedure}

\subsection{Diffractometer configuration}

All bulk texture measurements were conducted on a PANalytical X'pert-Pro Materials Research Diffractometer (MRD) goniometer (320 mm radius) equipped with a $\mathrm{Cu}$ tube operating in point focus mode at $40 \mathrm{kV}$ and $\sim 45 \mathrm{~mA}$. The incident beam optics comprised a polycapillary X-ray lens of $7 \mathrm{~mm}$ diameter and $\sim 0.3^{\circ}$ divergence angle. A square beam of $2 \times 2 \mathrm{~mm}^{2}$ was obtained by placing a knob adjustable crossed slit collimator in front of the lens. This beam size is significantly smaller than the lens diameter divided by $\sqrt{2}$ which ensures a well-shaped square beam and minimises any inhomogeneous lens transmission [4].

The diffracted beam optics comprised a $0.04^{\circ}$ soller slit, a parallel plate collimator $\left(0.27^{\circ}\right.$ acceptance angle), a flat crystal graphite monochromator and a PIXcel real time multiple strip (RTMS) detector operating in receiving slit mode.

\subsection{Bulk texture measurements on twinning induced plasticity (TWIP) steel}

A flat $25(l) \times 5(w) \times 1(t) \mathrm{mm}^{3}$ gage dog-bone-shaped sample of fully recrystallised $24 \mathrm{Mn}-3 \mathrm{Al}-$ 2Si-1Ni-0.06C (wt.\%) face centred cubic (fcc) austenitic TWIP steel was mechanically polished up to the colloidal silica stage and subjected to uniaxial tensile testing along the prior rolling direction, in speed control mode at $5 \mu \mathrm{m} \cdot \mathrm{s}^{-1}$. The details related to the earlier processing of the TWIP steel are given elsewhere [15].

The tensile test was interrupted at true strains of $0 \%, 7.2 \%, 20.9 \%, 32.3 \%$ and $48 \%$. Following each tensile test interruption, bulk texture measurements were conducted in the middle of the gauge length. After identifying the exact peak positions via a $\theta-2 \theta$ scan, the $\{111\},\{200\}$ and $\{220\}$ incomplete $\left(\alpha=0-85^{\circ}, \beta=0-360^{\circ}\right)$ pole figures were collected at $5^{\circ}$ intervals in step mode (counting time $=5 \mathrm{~s} /$ step). 
The background measurements were conducted at $\pm 2^{\circ}$ of a Bragg peak position. An austenitic stainless steel (ASS) texture-free reference sample was used to correct for the intensity loss in the $\{111\},\{200\}$ and $\{220\}$ incomplete pole figures of TWIP steel. The background and intensity loss correction measurements were conducted in continuous mode for a total counting time of $72 \mathrm{~s}$ at each tilt angle $(\alpha)$ such that one value of integrated intensity was returned after the sample rotated $(\beta)$ a full $360^{\circ}$ at each $\alpha$-angle.

The three sets of raw data files were imported to X'Pert Texture software to correct the incomplete pole figures for background and X-ray lens intensity. Following this, the normalized incomplete pole figures were exported to ResMat where the orientation distribution functions (ODFs) were calculated via series expansion. Since these ODFs do not account for intensity loss correction, they are hereafter termed as the uncorrected ODFs. An additional set of ODFs were calculated by correcting for the background, X-ray lens intensity and intensity loss and are hereafter referred to as experimental ODFs.

\subsection{Additional intensity loss scans on a set of texture-free reference samples}

Intensity loss correction measurements were collected for a range of Bragg angles $(\theta)$ using a set of texture-free reference samples comprising ASS (fcc), aluminium (Al, fcc), copper (Cu, fcc), ferritic stainless steel (FSS, body centred cubic (bcc)) and $\alpha$-titanium ( $\alpha$-Ti, hexagonal closed packed (hcp)) obtained from Labosoft S.C. and Bonet (Table 1). Similar to the method stated in Section 2.2, all measurements were conducted in continuous mode for a total counting time of $72 \mathrm{~s}$ at each tilt angle $(\alpha)$ such that one value of integrated intensity was returned after the sample rotated $(\beta)$ a full $360^{\circ}$ at each $\alpha$-angle.

For each Bragg angle, the intensity loss correction factor was computed via normalisation such that $U_{I}=I_{\alpha=0-85^{\circ}} / I_{\alpha=0^{\circ}}$. Figs. 1(a-e) depicts the experimental intensity loss correction curves of ASS (Fig. 1a), Al (Fig. 1b), Cu (Fig. 1c), FSS (Fig. 1d), $\alpha$-Ti (Fig. 1e).

Irrespective of the type of texture-free reference sample, the correction factors were then collated in a matrix in ascending order of Bragg angles and a linear fit [16] was performed for each tilt angle (Fig. 1f). Consequently, for a given goniometer configuration, these linear fits estimate the trend 
of intensity loss correction necessary for a given Bragg angle. In other words, the slope and intercept obtained from the linear fit at a given tilt angle are then used to empirically estimate (back-calculate) the intensity loss correction factor at any Bragg angle. The empirical intensity loss correction curves of ASS, Al, Cu, FSS, $\alpha$-Ti are superimposed on their experimental counterparts in Figs. 1(a-e). In almost all cases, close correspondence between the experimental and empirical trends is observed. The high $\mathrm{R}^{2-}$ values (>0.93) seen in Figs. 1(a-e) are attributed to the preservation of a linear relationship between the experimental and empirical intensity loss correction factors.

It follows that the above procedure enables us to generate correction curves for any Bragg angle when texture-free reference samples are not available for any alloy. To serve as a representative example, refer to the empirical intensity loss correction curves of the present TWIP steel at $0 \%$ strain (Fig. 2). Following this, the ODFs were calculated and are hereafter referred to as the empirical ODFs.

\section{Results and discussion}

The uncorrected, experimental and empirical $\phi_{2}=0^{\circ}, 45^{\circ}$ and $65^{\circ}$ ODF sections are shown in Figs. 3-5. The corresponding texture (T) and normalised difference $\left(T_{d}\right)$ indices are given in Table 2. While the uncorrected ODFs (Fig. 3) are qualitatively similar to their corrected counterparts (Figs. 4 and 5), quantitative differences are apparent such that: (i) the overall intensities of the uncorrected ODFs are higher as reflected in their higher $T$ indices, and (ii) the $T_{d}$ indices between the experimentaluncorrected ODFs are orders of magnitude higher than the experimental-empirical ODFs (Table 2). On the other hand, the empirical texture indices tend to be only slightly lower $(\Delta T=0.034 \pm 0.009)$ than the experimental ones such that the variation in normalised difference indices is almost negligible.

Consequently, the crystallographic texture results and trends are consistent in both, the experimental and the empirical ODFs (Figs. 4 and 5) as follows. At $0 \%$ strain (fully recrystallised condition), the texture is weak $(T=1.14-1.17)$ and comprises an $\alpha$-fibre (extending from Goss $(\{110\}\langle 001\rangle)$ to Rotated Goss $(\{011\}\langle 011\rangle)$ through Brass $(\{110\}\langle 112\rangle)$ as well as Cube $(\{001\}\langle 100\rangle$, Copper $(\{112\}\langle 111\rangle)$ and S $(\{123\}\langle 634\rangle)$ orientations; all of which were carried over from prior cold rolling [15]. Typical of fcc materials, uniaxial tension resulted in the development of the characteristic $\langle 111\rangle$ and $\langle 100\rangle$ double fibre texture parallel to the tensile loading axis. At $48 \%$ tensile strain 
(corresponding to the ultimate tensile strength), the maximum texture intensity lies on the $\langle 111\rangle$ fibre $(f(g)=7.5-7.7)$ compared to the $\langle 100\rangle$ fibre which records a maximum $f(g)=4.3-4.6$.

Based on the above, we can conclude that: (i) even when X-ray polycapillary lenses are used, an intensity loss correction should be applied when undertaking texture measurements using diffractometer configurations and sample sizes as described in Sections 2.1 and 2.2 and, (ii) the empirical methodology described in Section 2.3 can be readily applied to correct the pole figures of various engineering alloys when their texture-free reference samples are absent.

\section{Acknowledgments}

The authors acknowledge the use of the PANalytical X'Pert-PRO MRD texture goniometer purchased with financial support from the Australian Research Council (LE0989492).

\section{References}

[1] O. Engler, V. Randle, Introduction to texture analysis: Macrotexture, microtexture and orientation mapping, Second edition, Taylor \& Francis Group, LLC: CRC Press, USA, 2009, 86-105.

[2] K. Nagao, E. Kagami, X-ray thin film measurement techniques VII. Pole figure measurement, Rigaku J., 27 (2011) 6-14.

[3] E. Tenckhoff, Defocusing for the Schulz technique of determining preferred orientation, J. Appl. Phys., 41 (1970) 3944-3948.

[4] U. Welzel, M. Leoni, Use of polycapillary X-ray lenses in the X-ray diffraction measurement of texture, J. Appl. Crystallogr., 35 (2002) 196-206.

[5] W.P. Chernock, P.A. Beck, Analysis of certain errors in the X-ray reflection method for the quantitative determination of preferred orientations, J. Appl. Phys., 23 (1952) 341-345.

[6] R.A. Holt, J.E. Winegar, The effect of X-ray linewidth on the defocus correction in the Schulz reflection technique for texture measurement, J. Appl. Phys., 48 (1977) 3557-3559.

[7] L. Lahn, H.P. Hougardy, To the reliability of X-ray texture measurements: Important parameters, measuring strategies, scatter bands and comparative measurements with different systems, Mat.-wiss. u. Werkstofftech., 30 (1999) 77-86.

[8] G.B. Mitra, N.K. Misra, Background errors in X-ray diffraction parameters, Br. J. Appl. Phys., 17 (1966) 1319-1328.

[9] B. Gale, D. Griffiths, Influence of instrumental aberrations on the Schultz technique for the measurement of pole figures, Br. J. Appl. Phys., 11 (1960) 96-102.

[10] M. Ortiz, J.D. Hermida, A method for obtaining the complete pole figure with a single sample using the Schulz technique, Texture Cryst. Solids, 4 (1981) 159-169. 
[11] J.D. Hermida, Influence of the sample radius and irradiated zone width on the defocusing phenomenon in the Schulz technique, Mater. Sci. Eng., 56 (1982) 135-141.

[12] P. Scardi, S. Setti, M. Leoni, Multicapillary optics for materials science studies, Mater. Sci. Forum, 321-324 (2000) 162-167.

[13] M. Leoni, U. Welzel, P. Scardi, Polycapillary optics for materials science studies: Instrumental effects and their correction, J. Res. Natl. Inst. Stand. Technol., 109 (2004) 27-48.

[14] V.A. Kogan, J. Bethke, X-Ray optics for materials research, Mater. Sci. Forum, 278-281 (1998) 227235.

[15] A.A. Saleh, E.V. Pereloma, A.A. Gazder, Microstructure and texture evolution in a twinninginduced-plasticity steel during uniaxial tension, Acta Mater., 61 (2013) 2671-2691.

[16] Y.S. Liu, L. Depre, L. De Buyser, T.B. Wu, P. Van Houtte, Intensity correction in texture measurement of polycrystalline thin films by X-ray diffraction, Texture Microst., 35 (2003) 283-290. 


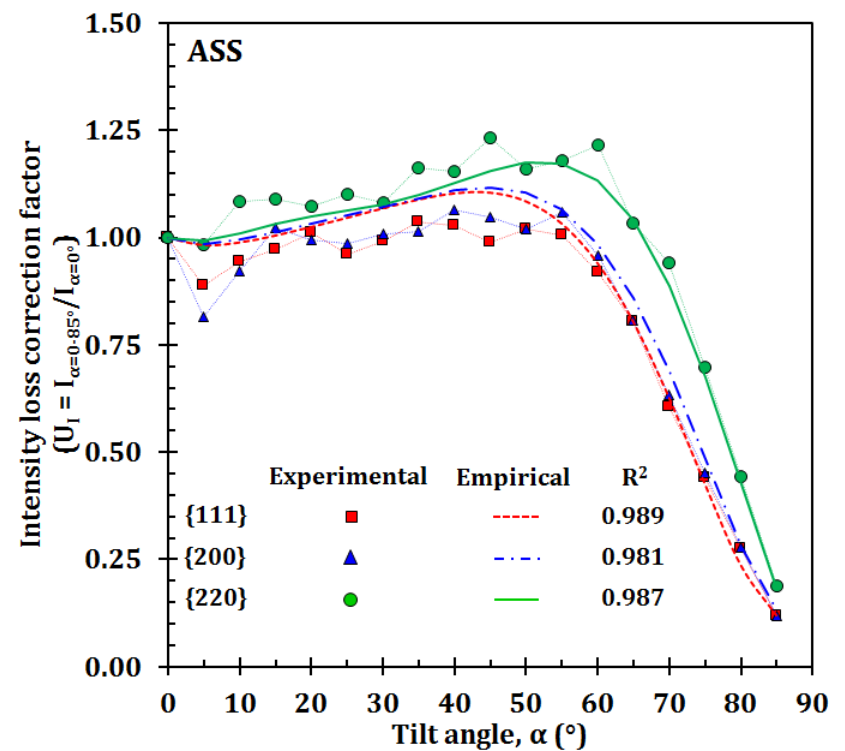

(a)

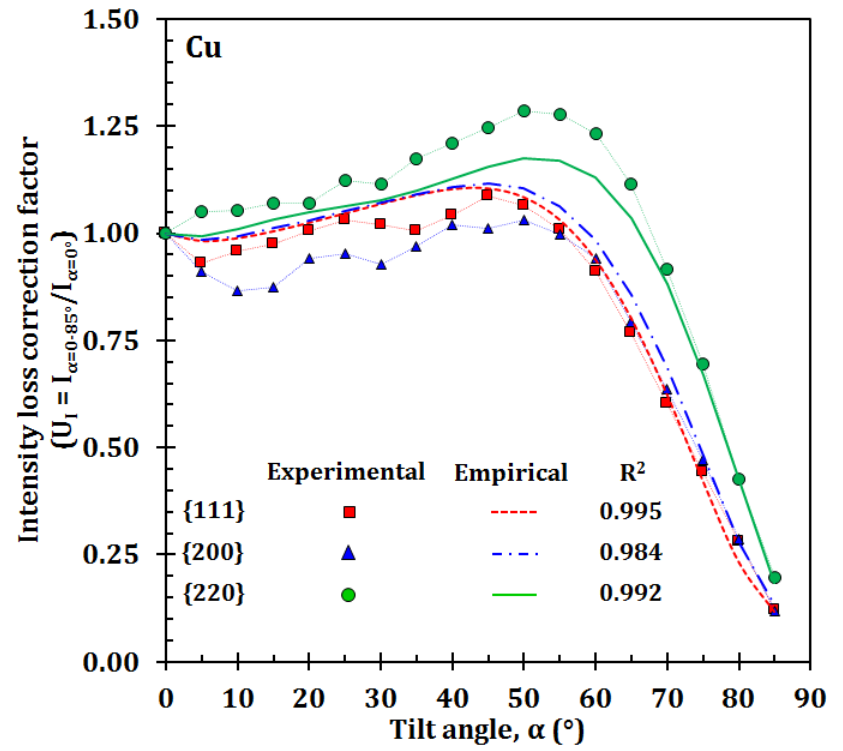

(c)

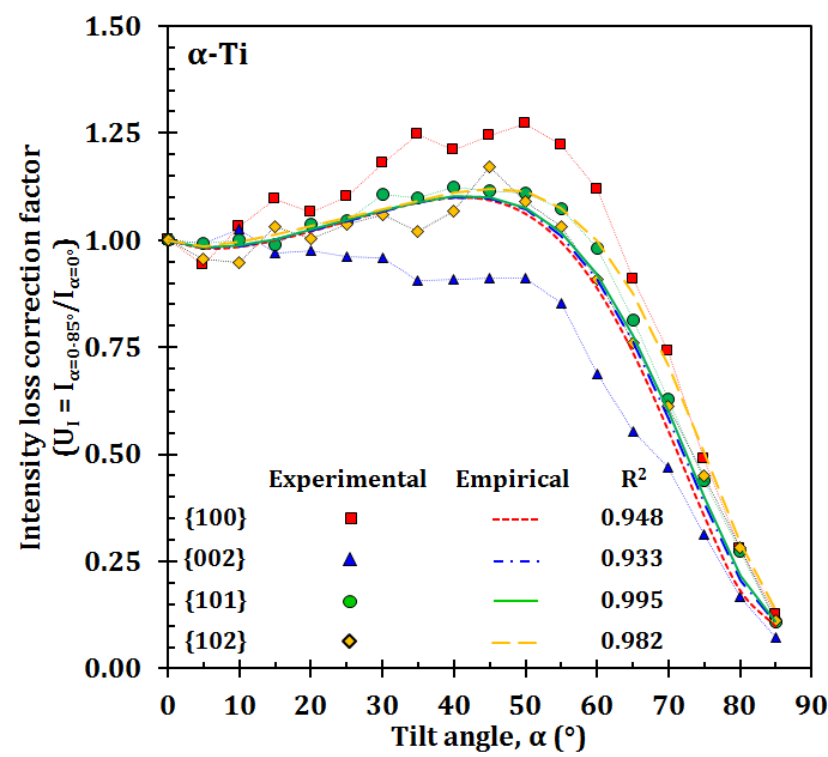

(e)

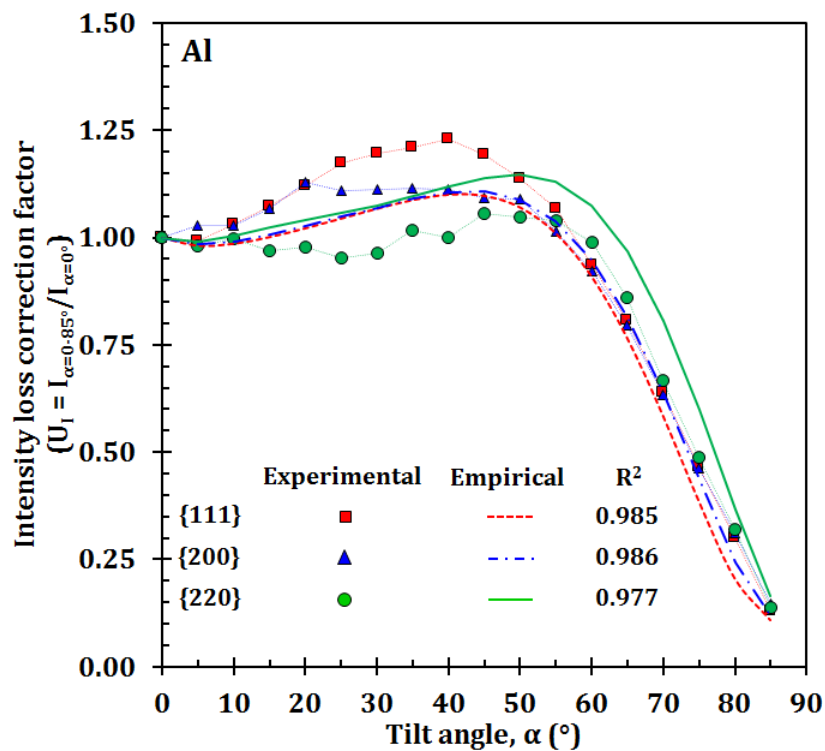

(b)

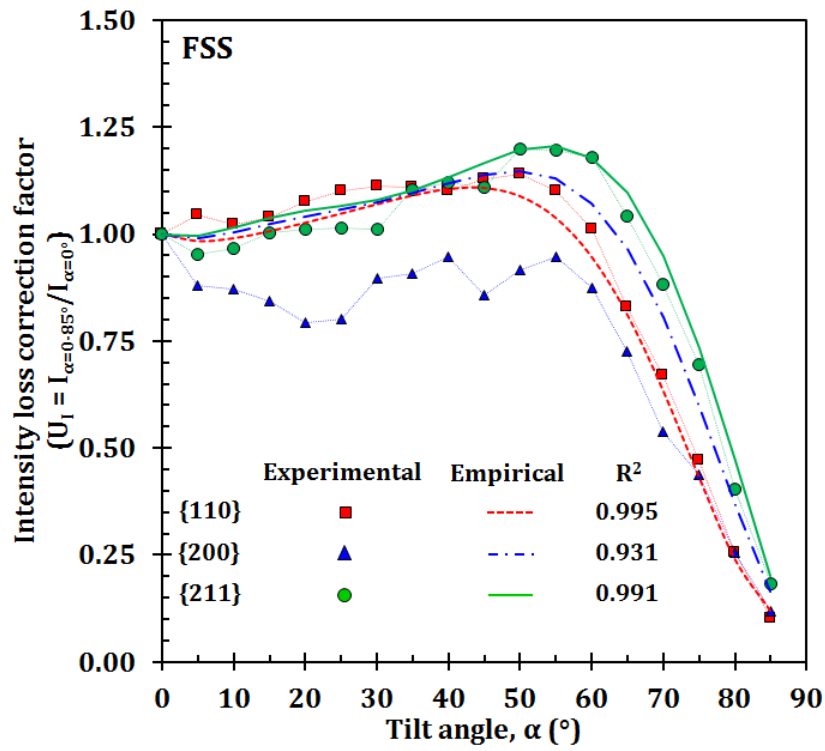

(d)

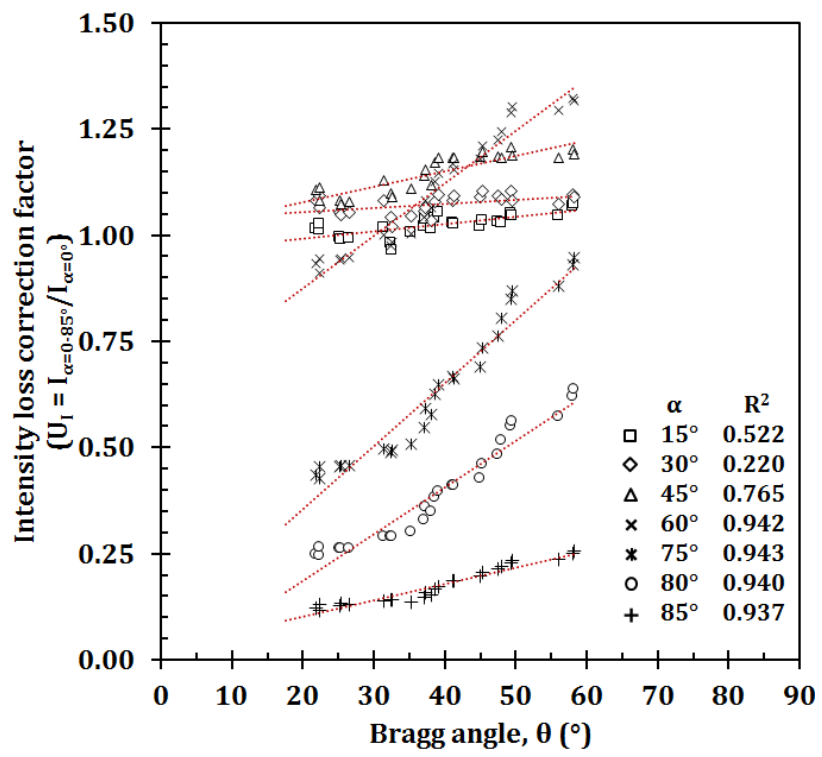

(f)

Fig. 1. The intensity loss correction factor $\left(\mathrm{U}_{\mathrm{I}}\right)$ as a function of (a-e) tilt and (f) Bragg angles. 


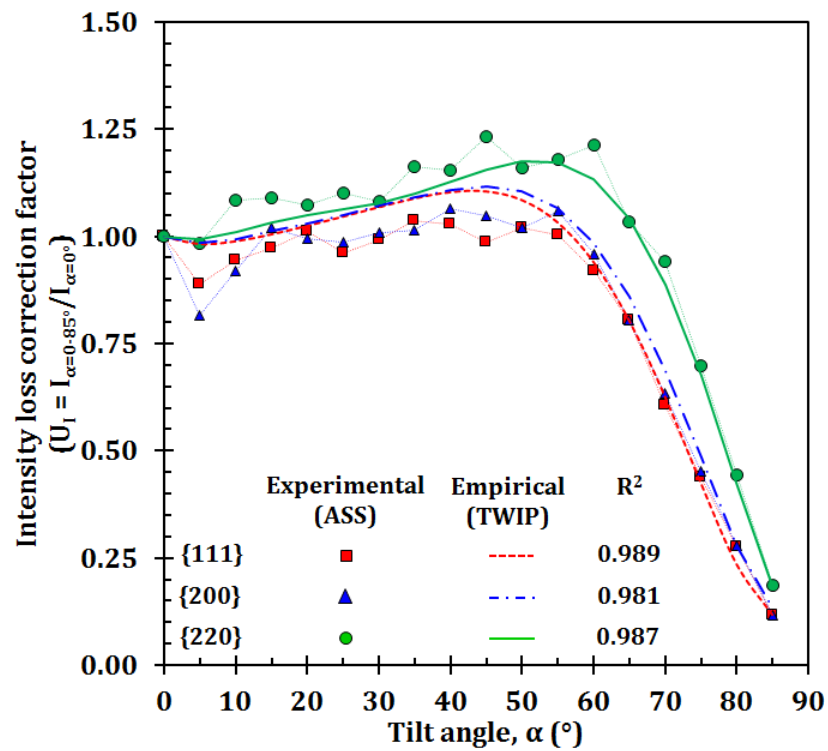

Fig. 2. The experimental and empirical intensity loss correction factors $\left(U_{I}\right)$ of the ASS texture-free reference sample and TWIP steel at $0 \%$ strain, respectively. 

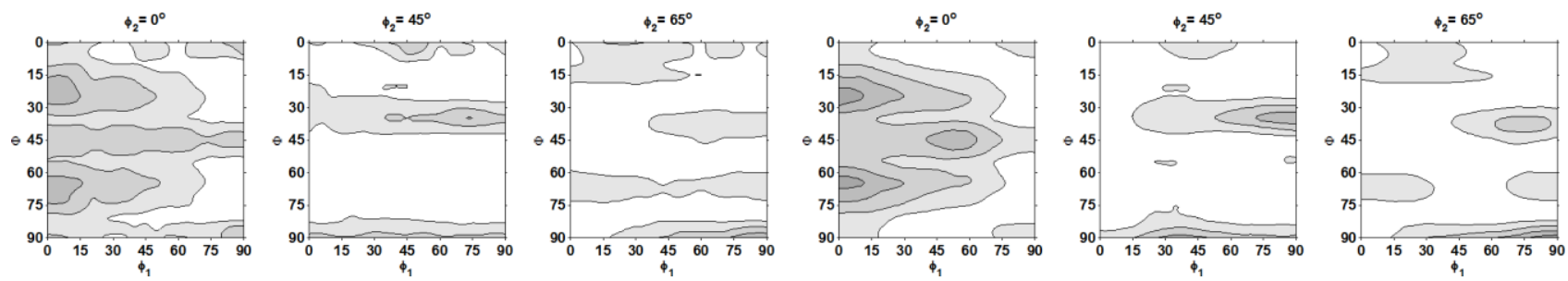

(a)
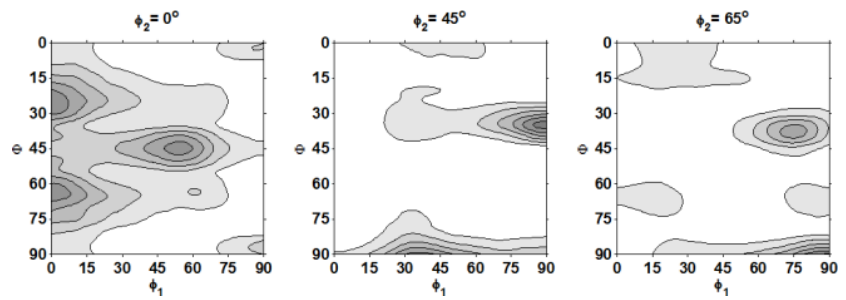

(c)

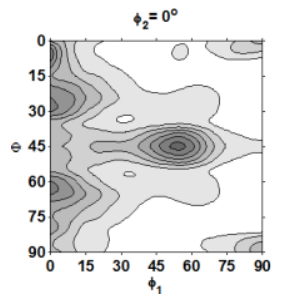

(b)
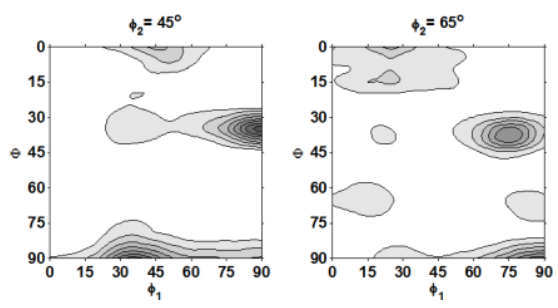

(d)
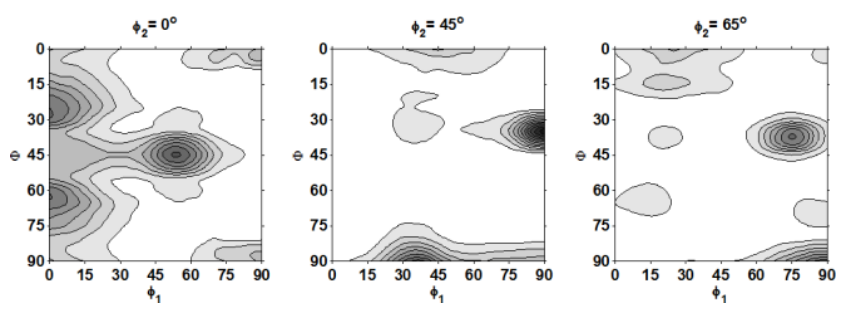

(e)

Fig. 3. Uncorrected (without intensity loss correction) $\phi_{2}=0^{\circ}, 45^{\circ}$ and $65^{\circ}$ ODF sections at tensile strains of (a) $0 \%$, (b) 7.2\%, (c) 20.9\%, (d) $32.3 \%$ and (e) $48 \%$. Contour levels $=1 \times$. 

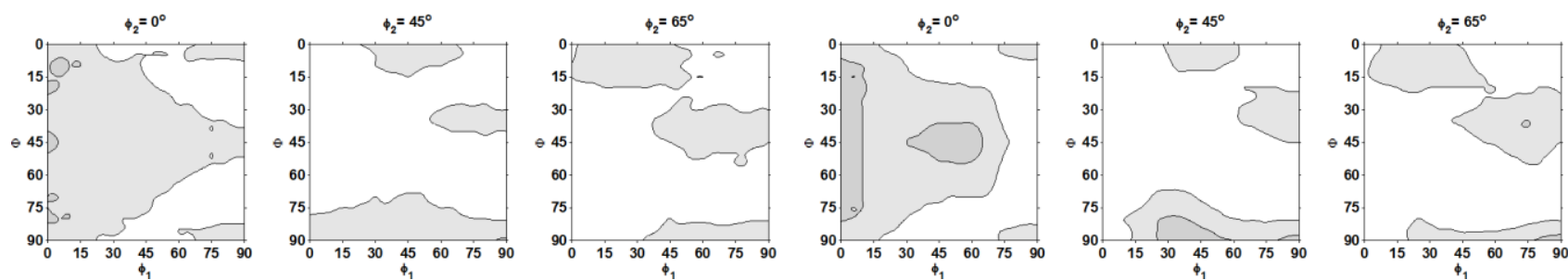

(a)
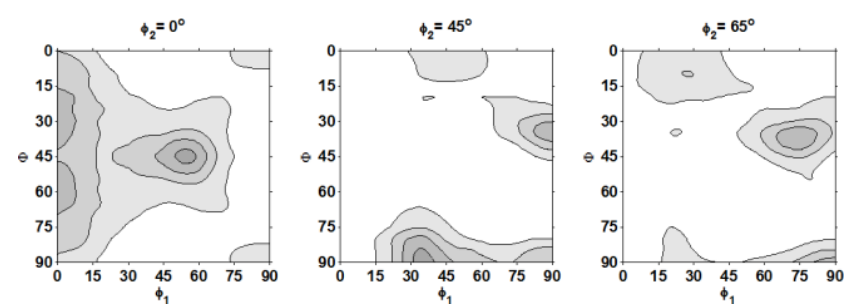

(c)

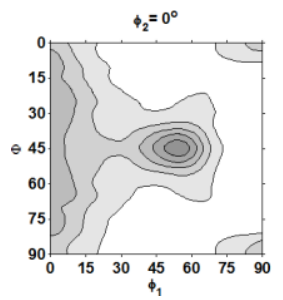

(b)

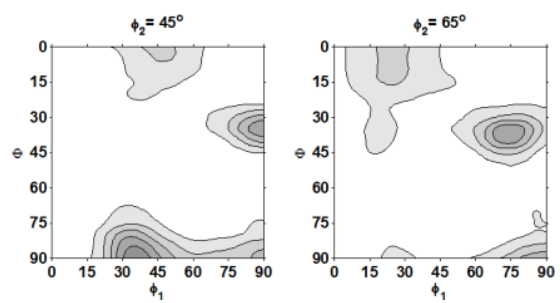

(d)
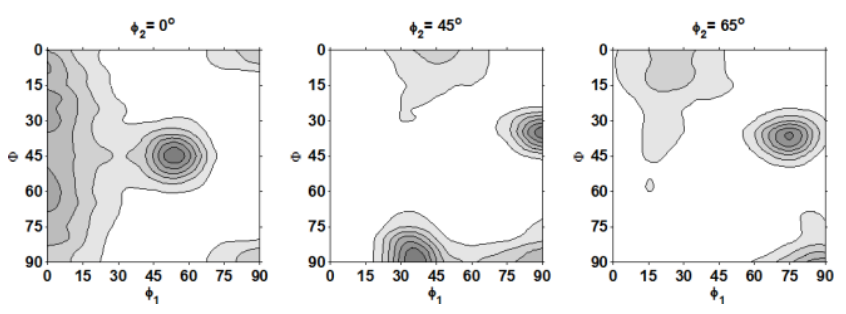

(e)

Fig. 4. Experimental (corrected using an ASS texture-free reference sample) $\phi_{2}=0^{\circ}, 45^{\circ}$ and $65^{\circ} \mathrm{ODF}$ sections at tensile strains of (a) 0\%, (b) 7.2\%, (c) 20.9\%, (d) 32.3\% and (e) 48\%. Contour levels = $1 \times$. 

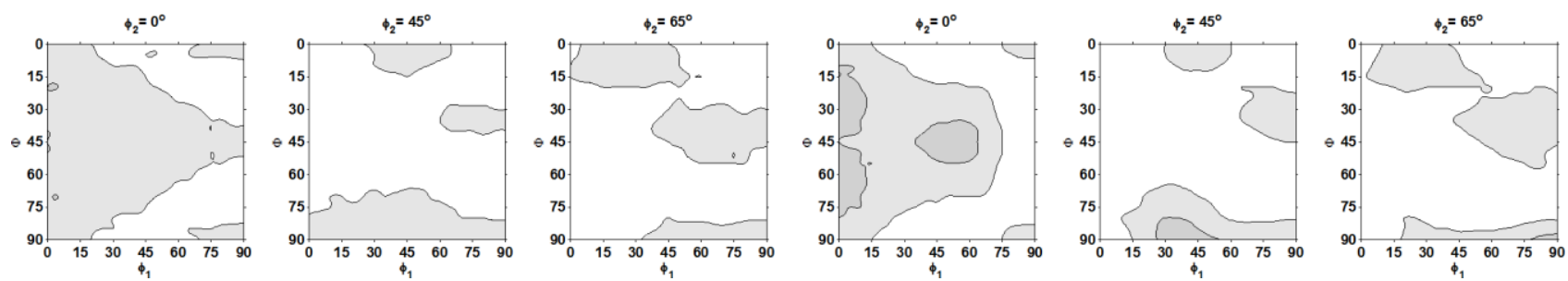

(a)
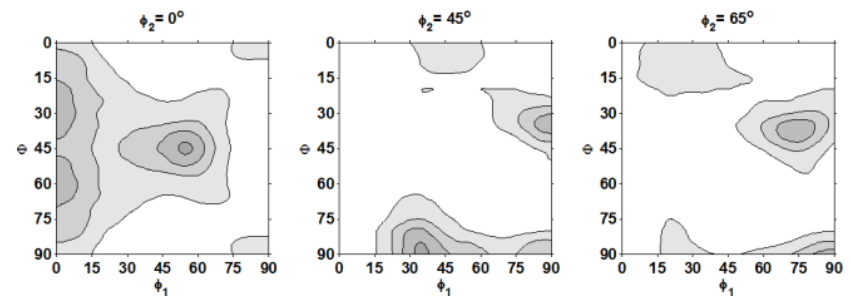

(c)

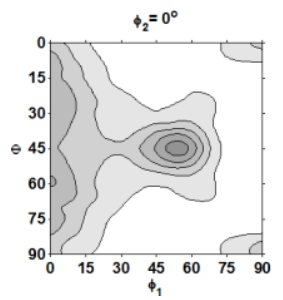

(b)

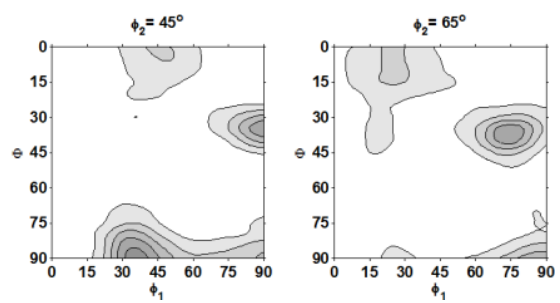

(d)
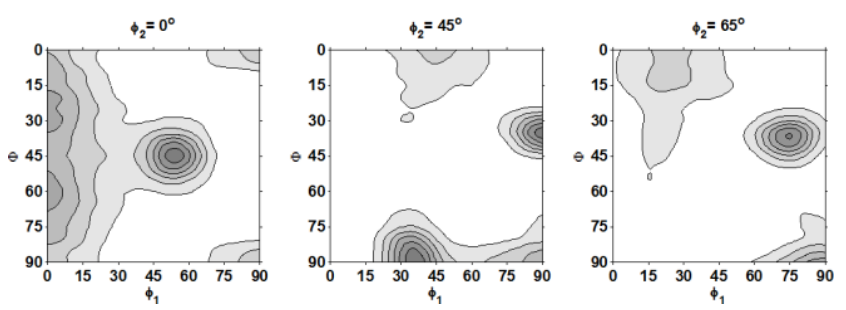

(e)

Fig. 5. Empirical (corrected using the empirical-TWIP curves in Fig. 2) $\phi_{2}=0^{\circ}, 45^{\circ}$ and $65^{\circ}$ ODF sections at tensile strains of (a) 0\%, (b) 7.2\%, (c) 20.9\%, (d) 32.3\% and (e) 48\%. Contour levels $=1 \times$. 
Table 1. Peak positions ( $2 \theta$ in degrees) for a $\mathrm{Cu}-\mathrm{K}_{\alpha} \mathrm{X}$-ray tube at which intensity correction factors were computed as a function of the tilt angle $(\alpha)$ for the set of texture-free reference samples obtained from Labosoft S.C. and Bonet. (The peak positions at which intensity loss correction factors were not computed are missing their $2 \theta$ values).

\begin{tabular}{cccc|cc|cc}
\hline fcc planes & ASS & Al & Cu & bcc planes & FSS & hcp planes & $\boldsymbol{\alpha}$-Ti \\
\hline$\{111\}$ & 43.61 & 38.48 & 43.33 & $\{110\}$ & 44.58 & $\{10 \overline{1} 0\} /\{100\}$ & 35.11 \\
$\{200\}$ & 50.79 & 44.74 & 50.47 & $\{200\}$ & 64.91 & $\{0002\} /\{002\}$ & 38.43 \\
$\{220\}$ & 74.69 & 65.12 & 74.17 & $\{211\}$ & 82.15 & $\{10 \overline{1} 1\} /\{101\}$ & 40.18 \\
$\{311\}$ & 90.65 & 78.25 & 89.99 & $\{220\}$ & 98.61 & $\{10 \overline{1} 2\} /\{102\}$ & 53.03 \\
$\{222\}$ & 95.93 & 82.46 & 95.14 & $\{310\}$ & 115.95 & $\{11 \overline{2} 0\} /\{110\}$ & 62.99 \\
$\{400\}$ & - & 99.06 & - & $\{222\}$ & 136.48 & $\{10 \overline{1} 3\} /\{103\}$ & 70.70 \\
$\{331\}$ & 138.24 & 112.03 & 136.50 & & & $\{20 \overline{2} 0\} /\{200\}$ & - \\
$\{420\}$ & 146.93 & 116.57 & 144.65 & & & $\{11 \overline{2} 2\} /\{112\}$ & 76.28 \\
$\{422\}$ & - & 137.46 & - & & & $\{20 \overline{2} 1\} /\{201\}$ & 77.39 \\
\hline
\end{tabular}

Table 2. The texture $(T)$ and normalised difference $\left(T_{d}\right)$ indices of the uncorrected, experimental and empirical ODFs as a function of tensile strain. The texture indices of the experimental ODFs were used as the reference to compute $T_{d}$.

\begin{tabular}{lccccc|ccccc}
\hline & \multicolumn{4}{c|}{ Texture index (T) } & \multicolumn{5}{c}{ Normalised difference index $\left(\boldsymbol{T}_{d}\right)$} \\
Strain (\%) & $\mathbf{0}$ & $\mathbf{7 . 2}$ & $\mathbf{2 0 . 9}$ & $\mathbf{3 2 . 3}$ & $\mathbf{4 8}$ & $\mathbf{0}$ & $\mathbf{7 . 2}$ & $\mathbf{2 0 . 9}$ & $\mathbf{3 2 . 3}$ & $\mathbf{4 8}$ \\
\hline Uncorrected & 1.48 & 1.55 & 1.93 & 2.43 & 2.75 & 0.1219 & 0.1215 & 0.0980 & 0.1007 & 0.0933 \\
Experimental & 1.17 & 1.24 & 1.61 & 1.93 & 2.33 & - & - & - & - & - \\
Empirical & 1.14 & 1.21 & 1.58 & 1.90 & 2.28 & 0.0013 & 0.0013 & 0.0009 & 0.0007 & 0.0008 \\
\hline
\end{tabular}

